\title{
ON CONVEXITY AND WEAK CLOSENESS FOR THE SET OF $\Phi$-SUPERHARMONIC FUNCTIONS
}

\author{
HONGWEI LOU
}

(Received 11 September 2001; revised 8 January 2003)

\author{
Communicated by A. H. Dooley
}

\begin{abstract}
Convexity and weak closeness of the set of $\Phi$-superharmonic functions in a bounded Lipschitz domain in $\mathbb{R}^{n}$ is considered. By using the fact of that $\Phi$-superharmonic functions are just the solutions to an obstacle problem and establishing some special properties of the obstacle problem, it is shown that if $\Phi$ satisfies $\Delta_{2}$-condition, then the set is not convex unless $\Phi(r)=C r^{2}$ or $n=1$. Nevertheless, it is found that the set is still weakly closed in the corresponding Orlicz-Sobolev space.
\end{abstract}

2000 Mathematics subject classification: primary 31C45; secondary $35 \mathrm{~J} 85$.

Keywords and phrases: convexity, weak closeness, superharmonic functions.

\section{Introduction}

Let $\Phi(\cdot)$ be a Young's function satisfying:

(S1) $\Phi(\cdot) \in C^{1}[0,+\infty) \cap C^{2}(0,+\infty)$;

(S2) $\Phi(0)=\Phi^{\prime}(0)=0$;

(S3) $\Phi^{\prime}(\cdot)$ increases strictly in $[0,+\infty)$, and $\lim _{r \rightarrow+\infty} \Phi^{\prime}(r)=+\infty$.

Denote $\varphi=\Phi^{\prime}$. Let $\psi=\varphi^{-1}$ be the inverse function of $\psi$ and

$$
\Psi(r) \triangleq \int_{0}^{r} \psi(s) d s, \quad \forall r \in \mathbb{R}^{+} \equiv[0,+\infty) .
$$

One can easily verify that $\Psi$ satisfies (S1)-(S3) too. We call $(\Phi, \Psi)$ a complementary pair in Young's sense. The well-known Young's inequality shows that

$$
r s \leq \Phi(r)+\Psi(s), \quad \forall r, s \in \mathbb{R}^{+},
$$

(C) 2003 Australian Mathematical Society $1446-7887 / 03 \$ A 2.00+0.00$ 
and the equality holds if and only if $s=\varphi(r)$.

In this paper, we will consider the set of $\Phi$-superharmonic functions $\mathscr{H}_{+}^{\Phi}(\Omega)$ (see (3.2) in Section 3 for the definition). We will prove that, if both $\Phi$ and $\Psi$ satisfy the $\Delta_{2}$-condition (see Lemma 2.1 (iv) in Section 2 for details), then $\mathscr{H}_{+}^{\Phi}(a, b)$ is convex. Consequently, it is weakly closed in $W_{0}^{1, \Phi}(a, b)$ since the strong closeness holds naturally. When $n \geq 2$, if $\Phi$ satisfies $\Delta_{2}$-condition and $\Omega$ is a bounded Lipschitz domain in Euclidean space $\mathbb{R}^{n}$, then $\mathscr{H}_{+}^{\Phi}(\Omega)$ is convex if and only if $\Phi(r)=C r^{2}$ for some positive constant $C$. Nevertheless, $\mathscr{H}_{+}^{\Phi}(\Omega)$ is still weakly closed in $W_{0}^{1, \Phi}(\Omega)$. The main idea to get the results is as follows: we use the fact that $\mathscr{H}_{+}^{\Phi}(\Omega)$ is the set of solutions for obstacle problems. From this we get many important properties of a $\Phi$-superharmonic function.

\section{Preliminary properties of Orlicz spaces}

In this section, we present some basic properties of Orlicz spaces. For further information about Orlicz spaces, see $[1,4,8]$.

First, let us recall the definition of Orlicz spaces $L^{\Phi}(\Omega), \mathscr{M}^{\Phi}(\Omega)$. Let $\Omega$ be a bounded domain in $\mathbb{R}^{n}$. We denote

$L^{\Phi}(\Omega) \triangleq\left\{v: \Omega \rightarrow \mathbb{R}\right.$ measurable $\mid \exists t>0$, such that $\left.\int_{\Omega} \Phi(t|v(x)|) d x<+\infty\right\}$,

equipped with the norm

$$
\|v\|_{L^{\bullet}(\Omega)} \equiv \inf \left\{t>0 \mid \int_{\Omega} \Phi\left(\frac{|v(x)|}{t}\right) d x \leq 1\right\},
$$

and

$$
\mathscr{M}^{\Phi}(\Omega) \triangleq\left\{v: \Omega \rightarrow \mathbb{B} \text { measurable } \mid \int_{\Omega} \Phi(t|v(x)|) d x<+\infty, \forall t>0\right\} .
$$

The following results can be found in [4].

LEMMA 2.1. Let $\Phi$ be Young's function satisfying (S1)-(S3), and $\Psi$ be defined by (1.1). Then

(i) $L^{\Phi}(\Omega)$ is a Banach space and $L^{\infty}(\Omega) \subset \mathscr{M}^{\Phi}(\Omega) \subseteq L^{\Phi}(\Omega) \subset L^{1}(\Omega)$.

(ii) $\mathscr{M}^{\Phi}(\Omega)$ is a Banach subspace of $L^{\Phi}(\Omega)$ and $\mathscr{M}^{\Phi}(\Omega)$ is separable.

(iii) $\left(\mathscr{M}^{\Psi}(\Omega)\right)^{*}=L^{\Phi}(\Omega)$, where $X^{*}$ denotes the dual space of a normed linear space $X$.

(iv) $\mathscr{M}^{\Phi}(\Omega)=L^{\Phi}(\Omega)$ if and only if $\Phi$ satisfies $\Delta_{2}$-condition (that is, there exist $\rho, \lambda>0$ such that $\Phi(2 r) \leq \lambda \Phi(r)$, for all $r \geq \rho)$. 
(v) $L^{\Phi}(\Omega)$ is reflexive if and only if both $\Phi$ and $\Psi$ satisfy $\Delta_{2}$-condition; if and only if $\Phi$ satisfies $\Delta_{2}$-condition, and $\Phi$ satisfies $\nabla_{2}$-condition (that is, there exist $\rho$, $l>1$ such that $\Phi(r) \leq \Phi(l r) /(2 l)$, for all $r \geq \rho)$.

Now, we recall the definition of Orlicz-Sobolev spaces. Denote

$$
W^{1, \Phi}(\Omega) \triangleq\left\{v: \Omega \rightarrow \mathbb{R} \mid v, D_{\alpha} v \in L^{\Phi}(\Omega), \alpha=1,2, \ldots, n\right\},
$$

equipped with the norm

$$
\|v\|_{W^{1, \Phi}(\Omega)} \equiv \sum_{\alpha=1}^{n}\left\|D_{\alpha} v\right\|_{L^{\Phi}(\Omega)}+\|v\|_{L^{\Phi}(\Omega)} .
$$

Let $W_{0}^{1, \Phi}(\Omega)$ be the closure of $C_{0}^{\infty}(\Omega)$ in $W^{1, \Phi}(\Omega)$. Denote

$$
\|v\|_{W_{0}^{1, \Phi}(\Omega)} \equiv\|\nabla v\|_{L^{\Phi}(\Omega)}, \quad \forall v \in W_{0}^{1, \Phi}(\Omega)
$$

Then, $\|\cdot\|_{W_{0}^{1 . \oplus}(\Omega)}$ is an equivalent norm to $\|\cdot\|_{W^{1 . \oplus}(\Omega)}$ in $W_{0}^{1, \Phi}(\Omega)$. Moreover, we can get the following properties by straightforward generalization of the proof of the same properties for ordinary Sobolev spaces.

LEMMA 2.2. Let $\Omega$ be a bounded domain, $\Phi$ be Young's function satisfying (S1)(S3).

(i) Suppose that $u \in W_{0}^{1, \Phi}(\Omega)$. Then $|u| \in W_{0}^{1, \Phi}(\Omega)$. Consequently, $u^{+} \equiv$ $\max (u, 0) \in W_{0}^{1, \Phi}(\Omega), u^{-} \equiv \max (-u, 0) \in W_{0}^{1, \Phi}(\Omega)$. Furthermore, if $u, v \in$ $W_{0}^{1, \Phi}(\Omega)$, then $u \wedge v \equiv \min (u, v) \in W_{0}^{1, \Phi}(\Omega)$.

(ii) Suppose that $\Omega$ is a Lipschitz domain. Then $W_{0}^{1, \Phi}(\Omega)=W_{0}^{1,1}(\Omega) \cap W^{1, \Phi}(\Omega)$.

If $L^{\Phi}(\Omega)$ is reflexive, then both $W^{1, \Phi}(\Omega)$ and $W_{0}^{1, \Phi}(\Omega)$ are reflexive, since they can be looked as closed subspaces of $\left(L^{\Phi}(\Omega)\right)^{n+1}$. In general, $W^{1, \Phi}(\Omega)$ (or $W_{0}^{1, \Phi}(\Omega)$ ) needs not necessary to be reflexive. Moreover, we do not know if $W^{1, \Phi}(\Omega)$ (or $\left.W_{0}^{1, \Phi}(\Omega)\right)$ is the dual space of a normed space, though $L^{\Phi}(\Omega)=\left(\mathscr{M}^{\Psi}(\Omega)\right)^{*}$ by Lemma 2.1 (iii). Thus, in general, a bounded series in $W^{1, \Phi}(\Omega)$ needs not necessary to have a subsequence converging weakly in $W^{1, \Phi}(\Omega)$. In some cases, we do not know if we can say weak* convergence. Nevertheless, we have:

LEMMA 2.3. Let $\Omega$ be a bounded Lipschitzdomain, $\Phi$ be Young's function satisfying (S1)-(S3), $u_{k}$ be bounded in $W^{1, \Phi}(\Omega)$. Then there exists a subsequence $u_{k_{j}}$, and a function $u \in W^{1, \Phi}(\Omega)$, such that $u_{k_{j}} \rightarrow u$, weakly in $W^{1.1}(\Omega)$, and

$$
\int_{\Omega} \Phi(|\nabla u|) d x \leq \liminf _{j \rightarrow+\infty} \int_{\Omega} \Phi\left(\left|\nabla u_{k_{j}}\right|\right) d x .
$$

Moreover, if $u_{k} \in W_{0}^{1, \Phi}(\Omega)$ for $k=1,2, \ldots$, then $u \in W_{0}^{1, \Phi}(\Omega)$. 
PROOF. Since $u_{k}$ is bounded in $W^{1, \Phi}(\Omega)$, then $u_{k}$ and $\left|\nabla u_{k}\right|$ are bounded in $L^{\Phi}(\Omega)$ (see Lemma 2.1 (i)). By Lemma 2.1 (ii) and (iii), $L^{\Phi}(\Omega)$ is the dual space of a separable Banach space $\mathscr{M}^{\Psi}(\Omega)$. Thus, we can choose a subsequence $u_{k_{j}}$ such that

$$
\begin{aligned}
u_{k_{j}} & \rightarrow u, \quad \text { weakly* in } L^{\Phi}(\Omega), \\
\nabla u_{k_{j}} & \rightarrow \vec{h}, \quad \text { weakly* in } L^{\Phi}\left(\Omega, \mathbb{R}^{n}\right) \equiv\left(L^{\Phi}(\Omega)\right)^{n} .
\end{aligned}
$$

Since $\left(\mathscr{M}^{\Psi}(\Omega)\right)^{*}=L^{\Phi}(\Omega)$ and $\left(L^{1}(\Omega)\right)^{*}=L^{\infty}(\Omega) \subset \mathscr{M}^{\Psi}(\Omega)$, we have

$$
\begin{aligned}
u_{k_{j}} & \rightarrow u, \quad \text { weakly in } L^{1}(\Omega), \\
\nabla u_{k_{j}} & \rightarrow \vec{h}, \quad \text { weakly in } L^{1}\left(\Omega, \mathbb{R}^{n}\right) .
\end{aligned}
$$

Then it follows that $\vec{h}=\nabla u$ and

$$
u_{k_{j}} \rightarrow u, \quad \text { weakly in } W^{1,1}(\Omega) .
$$

Combining with $u, \vec{h} \in L^{\Phi}(\Omega)$, we get $u \in W^{1, \Phi}(\Omega)$.

To prove (2.1), without loss of generality, we can suppose that

$$
\lim _{j \rightarrow+\infty} \int_{\Omega} \Phi\left(\left|\nabla u_{k_{j}}(x)\right|\right) d x=M<+\infty .
$$

On the other hand, by (2.2) and Mazur's theorem (see [7, page 120]), there exist $\alpha_{m, l} \geq 0, \sum_{l=1}^{k_{m}} \alpha_{m, l}=1$, such that

$$
\sum_{l=1}^{k_{m}} \alpha_{m, l} u_{k_{l+m}} \rightarrow u, \quad \text { strongly in } W^{1,1}(\Omega) .
$$

Thus, we can also suppose that

$$
\sum_{l=1}^{k_{m}} \alpha_{m, l} \nabla u_{k_{l+m}} \rightarrow \nabla u, \quad \text { a.e. } \Omega .
$$

By the convexity of $\Phi$, we have

$$
\int_{\Omega} \Phi\left(\left|\sum_{l=1}^{k_{m}} \alpha_{m, l} \nabla u_{k_{l+m}}(x)\right|\right) d x \leq \sum_{l=1}^{k_{m}} \alpha_{m, l} \int_{\Omega} \Phi\left(\left|\nabla u_{k_{l+m}}(x)\right|\right) d x .
$$

Noting that $\Phi \geq 0$ and $\Phi \in C[0,+\infty)$, by (2.3), (2.5)-(2.6) and Fatou's lemma, we get

$$
\int_{\Omega} \Phi(|\nabla u(x)|) d x \leq M,
$$

and therefore (2.1) follows.

Finally, if $u_{k} \in W_{0}^{1 . \Phi}(\Omega)$ for $k=1,2, \ldots$, then $u \in W_{0}^{1.1}(\Omega)$ by (2.2). Since we have obtained that $u \in W^{1 . \Phi}(\Omega)$, we get $u \in W_{0}^{1, \Phi}(\Omega)$ by Lemma 2.2 (ii). 


\section{3. $\Phi$-superharmonic functions and obstacle problem}

In the following sections we suppose that $\Phi$ satisfies $\Delta_{2}$-condition. Thus, $L^{\Phi}(\Omega)=$ $\mathscr{M}^{\Phi}(\Omega)$ by Lemma 2.1 (iv). Moreover,

$$
\int_{0}^{r} \varphi(s) d s=\Phi(r) \geq \frac{1}{\lambda} \Phi(2 r)=\frac{1}{\lambda} \int_{0}^{2 r} \varphi(s) d s, \quad \forall r \in(\rho,+\infty),
$$

where $\rho, \lambda>0$ are given in Lemma 2.1 (iv). Therefore

$$
r \varphi(r) \geq \Phi(r) \geq r \varphi(r) / \lambda, \quad r \in(\rho,+\infty),
$$

since $\varphi(\cdot)$ is increasing in $[0,+\infty)$. Then, for any $v, w \in W_{0}^{1, \Phi}(\Omega)$, by (3.1) and Young's inequality (1.2),

$$
\begin{aligned}
\left|\varphi(|\nabla v|) \frac{\nabla v}{|\nabla v|} \cdot \nabla w\right| & \leq \varphi(|\nabla v|)|\nabla w| \leq \Psi(\varphi(|\nabla v|))+\Phi(|\nabla w|) \\
& =|\nabla v| \varphi(|\nabla v|)-\Phi(|\nabla v|)+\Phi(|\nabla w|) \\
& \leq \max (\rho \varphi(\rho),(\lambda-1) \Phi(|\nabla v|))+\Phi(|\nabla w|) .
\end{aligned}
$$

Consequently, $\varphi(|\nabla v|) \nabla v \cdot \nabla w /|\nabla v|$ is integrable in $\Omega$.

Now, we denote by

$$
\mathscr{H}_{+}^{\Phi}(\Omega) \triangleq\left\{v \in W_{0}^{1, \Phi}(\Omega) \mid-\Delta_{\Phi} v \geq 0, \text { in } \Omega\right\}
$$

the set of all $\Phi$-superharmonic functions, where

$$
\Delta_{\Phi} v \triangleq \operatorname{div}\left(\varphi(|\nabla v|) \frac{\nabla v}{|\nabla v|}\right)
$$

and we say that $-\Delta_{\Phi} v \geq 0$ (in $\Omega$ ), if

$$
\int_{\Omega} \varphi(|\nabla v|) \frac{\nabla v}{|\nabla v|} \cdot \nabla w d x \geq 0, \quad \forall w \in W_{0}^{1, \Phi}(\Omega), w \geq 0, \text { a.e. } \Omega .
$$

An element of $\mathscr{H}_{+}^{\Phi}(\Omega)$ is called a $\Phi$-superharmonic function.

To study the set $\mathscr{H}_{+}^{\Phi}(\Omega)$, we consider the following obstacle problem:

PROBLEM (O). Let $y$ be a measurable function in $\Omega, \mathbb{K}(y) \triangleq\left\{v \in W_{0}^{1, \Phi}(\Omega) \mid v \geq\right.$ $y$, a.e. $\Omega\}$. Find a $u \equiv T_{\Phi}(y) \in \mathbb{K}(y)$, such that

$$
\int_{\Omega} \Phi(|\nabla u|) d x=\inf _{v \in \mathbb{K}(y)} \int_{\Omega} \Phi(|\nabla v|) d x .
$$


One can easily check that $\mathbb{K}(y)$ is convex and closed in $W_{0}^{1, \Phi}(\Omega)$. It may be empty. When $\mathbb{K}(y) \neq \emptyset$, the following lemma characterizes the solution of Problem $(0)$.

LEMMA 3.1. Let $\Omega$ be a bounded Lipschitz domain, y be a measurable function, $\mathbb{K}(y) \neq \emptyset, \Phi$ satisfy (S1)-(S3) and $\Delta_{2}$-condition. Then Problem $(\mathrm{O})$ has a unique solution $u \equiv T_{\Phi}(y)$. Moreover, $u$ is characterized by the following variational inequality:

$$
\int_{\Omega} \varphi(|\nabla u|) \frac{\nabla u}{|\nabla u|} \cdot \nabla(v-u) d x \geq 0, \quad \forall v \in \mathbb{K}(y) .
$$

ProOF. By Lemma 2.1 (iv), $L^{\Phi}(\Omega)=\mathscr{M}^{\Phi}(\Omega)$. Thus,

$$
0 \leq \int_{\Omega} \Phi(|\nabla v|) d x<+\infty, \quad \forall v \in \mathbb{K}(y) .
$$

Let $u_{k} \in \mathbb{K}(y)$ satisfy

$$
\lim _{k \rightarrow+\infty} \int_{\Omega} \Phi\left(\left|\nabla u_{k}\right|\right) d x=\inf _{v \in \mathbb{K}(y)} \int_{\Omega} \Phi(|\nabla v|) d x
$$

Then $\int_{\Omega} \Phi\left(\left|\nabla u_{k}\right|\right) d x \leq C$, for all $k=1,2, \ldots$, for some constant $C>0$. Since $\Phi(0)=0$ and $\Phi$ is convex,

$$
\int_{\Omega} \Phi\left(\frac{\left|\nabla u_{k}\right|}{C+1}\right) d x \leq \int_{\Omega} \frac{1}{C+1} \Phi\left(\left|\nabla u_{k}\right|\right) d x<1 .
$$

Therefore, $\left\|u_{k}\right\|_{w_{0}^{1 \cdot \bullet}(\Omega)} \leq C+1$, for all $k=1,2, \ldots$ Thus, by Lemma 2.3, we can suppose that $u_{k} \rightarrow u$, weakly in $W^{1,1}(\Omega)$, for some $u \in W_{0}^{1, \Phi}(\Omega)$, and

$$
\int_{\Omega} \Phi(|\nabla u|) d x \leq \liminf _{k \rightarrow+\infty} \int_{\Omega} \Phi\left(\left|\nabla u_{k}\right|\right) d x .
$$

On the other hand, it is easy to get $u \geq y$ from $u_{k} \geq y$. Consequently, $u \in \mathbb{K}(y)$, and it follows from (3.6) and (3.7) that

$$
\int_{\Omega} \Phi(|\nabla u|) d x=\inf _{v \in \mathbb{K}(y)} \int_{\Omega} \Phi(|\nabla v|) d x,
$$

that is, we get the existence of a solution. Since $\mathbb{K}(y)$ is convex and $G(\cdot)$ is strictly convex, such a solution must be unique.

Finally, to prove that $u \equiv T_{\Phi}(y)$ is characterized by (3.5), we modify the proof of Theorem 1.2 in [3, Chapter 1]. 
Let $u \equiv T_{\Phi}(y)$ be a solution of Problem (O) corresponding to $y$. Then, for all $v \in \mathbb{K}(y), \alpha \in(0,1)$, we have $u+\alpha(v-u) \in \mathbb{K}(y)$. Thus,

$$
0 \leq \frac{1}{\alpha}\left\{\int_{\Omega} \Phi(|\nabla u+\alpha(\nabla v-\nabla u)|) d x-\int_{\Omega} \Phi(|\nabla u|) d x\right\} .
$$

Let $\alpha \rightarrow 0^{+}$, we get (3.5).

On the other hand, suppose that $u \in \mathbb{K}(y)$ satisfies (3.5). Then, since $G(\cdot)$ is convex, we have

$$
\begin{aligned}
\left.\frac{1}{\alpha} \int_{\Omega}[\Phi(\mid \alpha \nabla v+(1-\alpha) \nabla u) \mid)-\Phi(|\nabla u|)\right] d x \\
\leq \frac{1}{\alpha} \int_{\Omega}[\Phi(\alpha|\nabla v|+(1-\alpha)|\nabla u|)-\Phi(|\nabla u|)] d x \\
\leq \frac{1}{\alpha} \int_{\Omega}[\alpha \Phi(|\nabla v|)+(1-\alpha) \Phi(|\nabla u|)-\Phi(|\nabla u|)] d x \\
\quad=\int_{\Omega}[\Phi(|\nabla v|)-\Phi(|\nabla u|)] d x, \quad \forall v \in \mathbb{K}(y) .
\end{aligned}
$$

Passing to the limit, we get

$$
\begin{aligned}
\int_{\Omega} \Phi(|\nabla v|) d x-\int_{\Omega} \Phi(|\nabla u|) d x & \geq \int_{\Omega} \varphi(|\nabla u|) \frac{\nabla u}{|\nabla u|} \cdot \nabla(v-u) d x \\
& \geq 0, \quad \forall v \in \mathbb{K}(y) .
\end{aligned}
$$

Therefore, $u$ is a solution of Problem $(O)$, and we get the proof.

Now, let us state a simple lemma before we establish the basic properties of $T_{\Phi}$.

LEMMA 3.2. Suppose $\phi(\cdot) \in C[0,+\infty), \phi(0)=0$, and $\phi$ increases strictly in $[0,+\infty)$. Then

$$
\left[\phi(|a|) \frac{a}{|a|}-\phi(|b|) \frac{b}{|b|}\right] \cdot(a-b) \geq 0, \quad \forall a, b \in \mathbb{R}^{m}
$$

and the equality holds if and only if $a=b$.

PROOF. Without loss of generality, we suppose that $a \neq 0, b \neq 0$. We have

$$
\begin{aligned}
& {\left[\phi(|a|) \frac{a}{|a|}-\phi(|b|) \frac{b}{|b|}\right] \cdot(a-b)} \\
& \quad=\phi(|a|)|a|+\phi(|b|)|b|-\phi(|a|) \frac{a \cdot b}{|a|}-\phi(|b|) \frac{a \cdot b}{|b|} \\
& \quad \geq \phi(|a|)|a|+\phi(|b|)|b|-\phi(|a|)|b|-\phi(|b|)|a| \\
& \quad=[\phi(|a|)-\phi(|b|)](|a|-|b|) \geq 0 .
\end{aligned}
$$


Moreover, in the second inequality, the equality holds if and only if $a \cdot b=|a||b|$, while in the last inequality, the equality holds if and only if $|a|=|b|$. Thus, (3.8) holds, and the equality holds if and only if $a=b$.

Now, we give some basic properties of $T_{\Phi}$.

LEMMA 3.3. Let $\Omega$ be a bounded Lipschitz domain, y be a measurable function, $\mathbb{K}(y) \neq \emptyset$, $\Phi$ satisfy (S1)-(S3) and $\Delta_{2}$-condition. Then

(i) $T_{\Phi}(y) \in \mathscr{H}_{+}^{\Phi}(\Omega)$.

(ii) $T_{\Phi}^{2}=T_{\Phi}$, that is, $T_{\Phi}\left(T_{\Phi}(y)\right)=T_{\Phi}(y)$.

(iii) $T_{\Phi}(y)=y$ if and only if $y \in \mathscr{H}_{+}^{\Phi}(\Omega)$.

(iv) Denote

$$
\begin{aligned}
\mathbb{K}_{+}(y) & \equiv\left\{v \in W^{1, \Phi}(\Omega) \mid v \geq y, \text { a.e. } \Omega\right\}, \quad \text { and. } \\
\mathscr{S}_{+}^{\Phi}(\Omega) & \equiv\left\{v \in W^{1, \Phi}(\Omega) \mid-\Delta v \geq 0, v \geq 0, \text { a.e. } \Omega\right\} .
\end{aligned}
$$

Then $T_{\Phi}(y)$ is the smallest element in $\mathbb{K}_{+}(y) \cap \mathscr{S}_{+}^{\Phi}(\Omega)$, that is, $T_{\Phi}(y) \in \mathbb{K}_{+}(y) \cap$ $\mathscr{S}_{+}^{\Phi}(\Omega)$ and $T_{\Phi}(y) \leq v$, a.e. $\Omega$, for all $v \in \mathbb{K}_{+}(y) \cap \mathscr{S}_{+}^{\Phi}(\Omega)$. In particular, $T_{\Phi}(y)$ is the smallest element in $\mathbb{K}_{+}(y) \cap \mathscr{H}_{+}^{\Phi}(\Omega)$ since $\mathscr{H}_{+}^{\Phi}(\Omega) \subset \mathscr{S}_{+}^{\Phi}(\Omega)$.

(v) Suppose $u_{1} \in \mathscr{H}_{+}^{\Phi}(\Omega), u_{2}, u_{2}, \ldots \in \mathscr{S}_{+}^{\Phi}(\Omega)$. Then $\underline{u} \equiv \inf _{k} u_{k} \in \mathscr{H}_{+}^{\Phi}(\Omega)$, and

$$
\int_{\Omega} \Phi(|\nabla \underline{u}|) d x \leq \int_{\Omega} \Phi\left(\left|\nabla u_{1}\right|\right) d x .
$$

PROOF. By Lemma 3.1, $u \equiv T_{\Phi}(y)$ exists and is unique.

(i) For any $v \in W_{0}^{1, \Phi}(\Omega), v \geq 0$, a.e. $\Omega$, we have $u+v \in \mathbb{K}(y)$. Replacing $v$ by $u+v$ in (3.5), we get

$$
\int_{\Omega} \varphi(|\nabla u|) \frac{\nabla u}{|\nabla u|} \cdot \nabla v d x \geq 0, \quad \forall v \in W_{0}^{1, \Phi}(\Omega), v \geq 0 .
$$

Therefore, $u \in \mathscr{H}_{+}^{\Phi}(\Omega)$.

(ii) Obviously, $u \in \mathbb{K}(u)$. Therefore, $\mathbb{K}(u) \neq \emptyset$. On the other hand, for $v \in \mathbb{K}(u)$, we have $v \in \mathbb{K}(y)$. Thus, by the definition of $u$ (see (3.4)),

$$
\int_{\Omega} \Phi(|\nabla u|) d x \leq \int_{\Omega} \Phi(|\nabla v|) d x, \quad \forall v \in \mathbb{K}(u) .
$$

Consequently, $u=T_{\Phi}(u)$. That is, $T_{\Phi}^{2}=T_{\Phi}$.

(iii) Let $T_{\Phi}(y)=y$. Then $y \in \mathscr{H}_{+}^{\Phi}(\Omega)$ by (i).

Now, suppose that $y \in \mathscr{H}_{+}^{\Phi}(\Omega)$. Then $y \in \mathbb{K}(y)$ and $\mathbb{K}(y) \neq \emptyset$. Since $-\Delta_{\Phi} y \geq 0$,

$$
\int_{\Omega} \varphi(|\nabla y|) \frac{\nabla y}{|\nabla y|} \cdot \nabla v d x \geq 0, \quad \forall v \in W_{0}^{1, \Phi}(\Omega), v \geq 0 .
$$


For any $v \in \mathbb{K}(y)$, we have $v-y \geq 0$, a.e. $\Omega$, and $v-y \in W_{0}^{1, \Phi}(\Omega)$. Thus,

$$
\int_{\Omega} \varphi(|\nabla y|) \frac{\nabla y}{|\nabla y|} \cdot \nabla(v-y) d x \geq 0, \quad \forall v \in \mathbb{K}(y) .
$$

Therefore, $y=T_{\Phi}(y)$ by Lemma 3.1.

(iv) Suppose $v \in \mathbb{K}_{+}(y) \cap \mathscr{S}_{+}^{\Phi}(\Omega)$. Then $v \wedge u \in W_{0}^{1,1}(\Omega)$ since $W^{1, \Phi}(\Omega) \subset$ $W^{1,1}(\Omega)$. Moreover,

$$
\nabla(v \wedge u)(x)=\left\{\begin{array}{ll}
\nabla v(x), & \text { if } v(x)<u(x) ; \\
\nabla u(x), & \text { if } v(x) \geq u(x),
\end{array} \text { a.e. } \Omega .\right.
$$

It follows that $v \wedge u \in W^{1, \Phi}(\Omega)$ since $v, u \in W^{1, \Phi}(\Omega)$. Consequently, $v \wedge u \in W_{0}^{1, \Phi}(\Omega)$ by Lemma 2.2 (ii). Thus, $v \wedge u \in \mathbb{K}(y)$. By Lemma 3.1, we get

$$
\int_{\Omega} \varphi(|\nabla u|) \frac{\nabla u}{|\nabla u|} \cdot(\nabla(v \wedge u)-\nabla u) d x \geq 0 .
$$

On the other hand, since $-\Delta_{\Phi} v \geq 0$, and $u-(v \wedge u) \geq 0, u-(v \wedge u) \in W_{0}^{1, \Phi}(\Omega)$, we get (see (3.3))

$$
\int_{\Omega} \varphi(|\nabla v|) \frac{\nabla v}{|\nabla v|} \cdot(\nabla u-\nabla(v \wedge u)) d x \geq 0 .
$$

Combining with (3.11), we have

$$
\int_{\Omega}\left[\varphi(|\nabla u|) \frac{\nabla u}{|\nabla u|}-\varphi(|\nabla v|) \frac{\nabla v}{|\nabla v|}\right] \cdot(\nabla u-\nabla(v \wedge u)) d x \leq 0
$$

Then, by (3.10),

$$
\int_{(u>v)}\left[\varphi(|\nabla u|) \frac{\nabla u}{|\nabla u|}-\varphi(|\nabla v|) \frac{\nabla v}{|\nabla v|}\right] \cdot(\nabla u-\nabla v) d x \leq 0 .
$$

Therefore, by Lemma 3.2, $\nabla u=\nabla v$, a.e. $\{u>v\}$, that is (see (3.10)), $\nabla u=\nabla(v \wedge u$ ), a.e. $\Omega$. Consequently, there exists a constant $C$, such that $u=v \wedge u+C$, a.e. $\Omega$. Since $u, v \wedge u \in W_{0}^{1, \Phi}(\Omega)$, we have $C=0$. Thus, $u=v \wedge u$, a.e. $\Omega$, that is, $u \leq v$, a.e. $\Omega$. On the other hand, it is easy to prove that any $v \in \mathscr{H}_{+}^{\Phi}(\Omega)$ satisfies $v \geq 0$, a.e. $\Omega$. Thus $\mathscr{H}_{+}^{\Phi}(\Omega) \subset \mathscr{S}_{+}^{\Phi}(\Omega)$. Therefore, $T_{\Phi}(y)$ is also the smallest member in $\mathbb{K}_{+}(y) \cap \mathscr{H}_{+}^{\Phi}(\Omega)$ since $T_{\Phi}(y) \in \mathbb{K}_{+}(y) \cap \mathscr{H}_{+}^{\Phi}(\Omega)$.

(v) Since $0 \leq \underline{u} \leq u_{1}, \mathbb{K}(\underline{u}) \neq \emptyset$. Thus, $T_{\Phi}(\underline{u})$ uniquely exists. By (iv) and noting that $u_{k} \in \mathbb{K}_{+}(\underline{u}) \cap \mathscr{S}_{+}^{\Phi}(\Omega)$, we have $T_{\Phi}(\underline{u}) \leq u_{k}$, a.e. $\Omega$. Thus,

$$
T_{\Phi}(\underline{u}) \leq \underline{u} \equiv \inf _{k} u_{k}, \quad \text { a.e. } \Omega .
$$

On the other hand, $T_{\Phi}(\underline{u}) \geq \underline{u}$ by the definition of $T_{\Phi}$. Therefore, $\underline{u}=T_{\Phi}(\underline{u}) \in$ $\mathscr{H}_{+}^{\Phi}(\Omega)$. Finally, since $u_{1} \in \mathbb{K}(\underline{u})$, we get (3.9) from (3.4). 


\section{Convexity of $\mathscr{H}_{+}^{\Phi}(\Omega)$}

First, we establish the following lemma.

LEMMA 4.1. Let $\Omega$ be a bounded domain in $\mathbb{R}^{n}, \Phi$ satisfy (S1)-(S3), $\Delta_{2}$-condition and $\nabla_{2}$-condition. Let $W^{-1, \Psi}(\Omega)$ be the dual space of $W_{0}^{1, \Phi}(\Omega)$. Let $\nu \in W^{-1, \Psi}(\Omega)$ and $\nu \geq 0$ in $\Omega$ in the distribution sense. Then, there exists a sequence $v_{k} \in C^{\infty}(\bar{\Omega})$, such that $v_{k} \geq 0$ and

$$
v_{k} \rightarrow \nu, \quad \text { strongly in } W^{-1, \Psi}(\Omega) .
$$

Proof. By the assumptions and Lemma $2.1(\mathrm{v}), W_{0}^{1, \Phi}(\Omega)$ is reflexive. Consequently, as the dual space of $W_{0}^{1, \Phi}(\Omega), W^{-1, \Psi}(\Omega)$ is reflexive.

Let $\nu \in W^{-1, \Psi}(\Omega)$ and $\nu \geq 0$ in $\Omega$ in the distribution sense. According to [5, Chapter 1 , Theorem V], $v$ is a nonnegative measure in $\Omega$. For $k=1,2, \ldots$, denote $\Omega_{k} \equiv\{x \in \Omega \mid d(x, \partial \Omega) \geq 1 / k\}$, where $d(x, \partial \Omega) \equiv \inf _{y \in \partial \Omega}|x-y|$. Let $v_{k} \equiv \nu\left\lfloor\Omega_{k}\right.$ be the restriction of $v$ in $\Omega_{k}$, that is, $\nu_{k}(A) \equiv v\left(\Omega_{k} \cap A\right)$ for any $A \subseteq \Omega$. Then, $\nu_{k}$ is a nonnegative measure in $\Omega$ (see [6, Chapter 1], for example). Moreover, for any $w \in C_{c}^{\infty}(\Omega)$, we have

$$
\left|\left\langle v_{k}, w\right\rangle\right| \leq\left\langle v_{k},|w|\right\rangle \leq\langle\nu,|w|\rangle \leq\|\nu\|_{W^{-1, \psi}(\Omega)}\|w\|_{W_{0}^{1, \Phi}(\Omega)} .
$$

Consequently, for any $w \in W_{0}^{1, \Phi}(\Omega)$, we can define $\left\langle v_{k}, w\right\rangle$ by choosing $w_{j} \in C_{c}^{\infty}(\Omega)$ converging strongly in $W_{0}^{1, \Phi}(\Omega)$ and defining

$$
\left\langle\nu_{k}, w\right\rangle \triangleq \lim _{j \rightarrow+\infty}\left\langle\nu_{k}, w_{j}\right\rangle .
$$

By (4.2),

$$
\left\langle v_{k}, w\right\rangle \leq\|v\|_{W^{-1, \psi_{(}}(\Omega)}\|w\|_{W_{0}^{1, \Phi}(\Omega)}, \quad \forall w \in W_{0}^{1, \Phi}(\Omega),
$$

that is, $\nu_{k} \in W^{-1, \psi}(\Omega)$ and

$$
\left\|v_{k}\right\| w_{-1, \psi(\Omega)} \leq\|\nu\|_{W^{-1 . \psi}(\Omega)} .
$$

On the other hand, we have $\left\langle\nu_{k}, w\right\rangle \rightarrow\langle\nu, w\rangle$, for all $w \in C_{c}^{\infty}(\Omega)$. Combining the above with (4.3), we can easily get $\left\langle v_{k}, w\right\rangle \rightarrow\langle\nu, w\rangle$, for all $w \in W_{0}^{1, \Phi}(\Omega)$, that is, $\nu_{k} \rightarrow \nu$, weakly in $W^{-1, \Psi}(\Omega)$.

By Mazur's Theorem, there exist $\alpha_{k, j} \geq 0, \sum_{j=1}^{N_{k}} \alpha_{k, j}=1$, such that $\tilde{v}_{k} \equiv$ $\sum_{j=1}^{N_{k}} \alpha_{k, j} \nu_{j} \rightarrow \nu$, strongly in $W^{-1, \Psi}(\Omega)$. For any $l \geq N_{k}$, we have

$$
\begin{aligned}
\left|\left\langle\nu_{l}-\nu, w\right\rangle\right| & \leq\left\langle\nu-v_{l},|w|\right\rangle \leq\left\langle\nu-\tilde{v}_{k},|w|\right\rangle \\
& \leq\left\|\nu-\tilde{v}_{k}\right\| w^{-1 . \psi}(\Omega)\|w\|_{w_{0}^{1 . \Phi}(\Omega)}, \quad \forall w \in W_{0}^{1, \Phi}(\Omega) .
\end{aligned}
$$


Thus, $\left\|\nu_{l}-v\right\|_{W^{-1, \psi}(\Omega)} \leq\left\|\tilde{\nu}_{k}-v\right\|_{W^{-1, \psi}(\Omega)}$, if $l \geq N_{k}$. Consequently,

$$
\nu_{k} \rightarrow \nu, \quad \text { strongly in } W^{-1, \Psi}(\Omega) \text {. }
$$

Let

$$
\eta(x) \equiv \begin{cases}\tau \exp \left[-1 /\left(1-|x|^{2}\right)\right], & \text { if }|x|<1 \\ 0, & \text { if }|x| \geq 1,\end{cases}
$$

where $\tau>0$ is chosen such that $\int_{\mathbb{R}^{n}} \eta(x) d x=1$. Let $\zeta_{k} \in C_{c}^{\infty}\left(\mathbb{R}^{n}\right)$ satisfy $\zeta_{k}(x)=1$, in $\Omega_{k}$, and $\zeta_{k}(x)=0$, in $\mathbb{R}^{n} \backslash \Omega_{2 k}$. Then $\zeta_{k} \nu_{k}$ can be looked as a distribution and a nonnegative measure in $\mathbb{R}^{n}$. In fact, $\zeta_{k} \nu_{k}=v_{k}$, in $\Omega$. For $j=1,2, \ldots$, set $\eta_{j}(x)=j \eta(j x)$, and $\hat{v}_{k j} \equiv\left(\zeta_{k} \nu_{k}\right) * \eta_{j}$ (for the definition of convolution of generalized function, see [2]). Obviously, $\hat{v}_{k j}(x) \geq 0$. When $j \geq 12 k$, we have $\hat{v}_{k j}(x)=0$, for all $x \notin \Omega_{3 k}$. Then, it is easy to prove that $\hat{v}_{k j} \in C_{\mathrm{c}}^{\infty}(\Omega)$ (if $j \geq 12 k$ ), and (as $j \rightarrow+\infty$ )

$$
\hat{v}_{k j} \rightarrow v_{k}, \quad \text { weakly in } W^{-1, \Psi}(\Omega) \text {. }
$$

Consequently, by Mazur's Theorem, we have $\tilde{v}_{k j} \in C^{\infty}(\bar{\Omega})$, such that $\tilde{v}_{k j} \geq 0$, and (as $j \rightarrow+\infty) \tilde{\nu}_{k j} \rightarrow v_{k}$, strongly in $W^{-1, \Psi}(\Omega)$. Thus, combining with (4.4), we have $j_{k} \geq 1$, such that $v_{k} \equiv \tilde{v}_{k j_{k}} \rightarrow v$, strongly in $W^{-1, \psi}(\Omega)$. Thus, we get the proof.

Now, we give a result in case $n=1$.

THEOREM 4.2. Let $a<b, \Phi$ satisfy (S1)-(S3), $\Delta_{2}$-condition and $\nabla_{2}$-condition. Then $u \in \mathscr{H}_{+}^{\Phi}(a, b)$ if and only if $u \in W_{0}^{1, \Phi}(a, b)$, and $-u^{\prime \prime} \geq 0$, in $(a, b)$. Consequently, $\mathscr{H}_{+}^{\Phi}(a, b)$ is convex.

PROOF. We give a sketch of the proof.

Let $u \in \mathscr{H}_{+}^{\Phi}(a, b)$. Then $v \equiv-\Delta_{\Phi} u \in W^{-1, \Psi}(a, b)$, and $v \geq 0$ in the distribution sense. By Lemma 4.1 , there exists $v_{j} \in C^{\infty}(\bar{\Omega})$, such that $v_{j} \geq 0$, and

$$
v_{j} \rightarrow \nu, \quad \text { strongly in } W^{-1, \Psi}(a, b) .
$$

Let $u_{j} \in W_{0}^{1, \Phi}(a, b)$ be the unique solution of the following equation:

$$
\left\{\begin{array}{l}
-\left[\varphi\left(\sqrt{1 / j^{2}+\left|u_{j}^{\prime}\right|^{2}}\right) u_{j}^{\prime} / \sqrt{1 / j^{2}+\left|u_{j}^{\prime}\right|^{2}}\right]^{\prime}=v_{j}, \quad \text { in }(a, b) ; \\
u_{j}(a)=u_{j}(b)=0 .
\end{array}\right.
$$

Then $u_{j} \in C^{\infty}(a, b)$, and $u_{j}$ is bounded in $W_{0}^{1, \Phi}(a, b)$. Since $W_{0}^{1, \Phi}(a, b)$ is reflexive, we can suppose that $u_{j} \rightarrow \tilde{u}$, weakly in $W_{0}^{1, \Phi}(a, b)$. Similarly as in Lemma 3.1 , it follows from (4.5) that for all $v \in W_{0}^{1, \Phi}(a, b)$,

$$
\int_{a}^{b} \Phi\left(\sqrt{1 / j^{2}+\left|u_{j}^{\prime}\right|^{2}}\right) d x-\left\langle v_{j}, u_{j}\right\rangle \leq \int_{a}^{b} \Phi\left(\sqrt{1 / j^{2}+\left|v^{\prime}\right|^{2}}\right) d x-\left\langle v_{j}, v\right\rangle .
$$


Passing to the limit (see the proof of (2.1)), we get

$$
\int_{a}^{b} \Phi\left(\left|\tilde{u}^{\prime}\right|\right) d x-\langle\nu, \tilde{u}\rangle \leq \int_{a}^{b} \Phi\left(\left|v^{\prime}\right|\right) d x-\langle\nu, v\rangle, \quad \forall v \in W_{0}^{1, \Phi}(a, b),
$$

that is,

$$
\left\{\begin{array}{l}
-\Delta_{\Phi} \tilde{u}=\nu, \\
\tilde{u}(a)=\tilde{u}(b)=0 .
\end{array}\right.
$$

Therefore, $\tilde{u}=u$. Consequently, $u_{j} \rightarrow u$, weakly in $W_{0}^{1, \Phi}(a, b)$.

Since $u_{j} \in C^{\infty}(a, b)$, by (4.5),

$$
-\left\{\frac{\varphi^{\prime}\left(\sqrt{1 / j^{2}+\left|u_{j}^{\prime}\right|^{2}}\right)\left|u_{j}^{\prime}\right|^{2}}{\sqrt{1 / j^{2}+\left|u_{j}^{\prime}\right|^{2}}}+\frac{\varphi\left(\sqrt{1 / j^{2}+\left|u_{j}^{\prime}\right|^{2}}\right)}{j^{2}\left(1 / j^{2}+\left|u_{j}^{\prime}\right|^{2}\right)^{3 / 2}}\right\} u_{j}^{\prime \prime}=v_{j} \geq 0, \quad \text { in }(a, b) .
$$

Thus, $-u_{j}^{\prime \prime} \geq 0$, in $(a, b)$. Passing to the limit, we get $-u^{\prime \prime} \geq 0$, in $(a, b)$. Similarly, if $u \in W_{0}^{1, \Phi}(a, b)$, and $-u^{\prime \prime} \geq 0$, in $(a, b)$, then we can prove that $u \in \mathscr{H}_{+}^{\Phi}(a, b)$.

When $n \geq 2$, we have:

THEOREM 4.3. Let $\Omega$ be a bounded Lipschitz domain. Suppose that $n \geq 2, \Phi$ satisfies (S1)-(S3) and $\Delta_{2}$-condition. Then $\mathscr{H}_{+}^{\Phi}(\Omega)$ is convex if and only if $\Phi(r) \equiv$ $C r^{2}$ for some positive constant $C$.

PRoOF. If $\Phi(r) \equiv C r^{2}$, then $\Delta_{\Phi}=2 C \Delta$. Consequently, $\mathscr{H}_{+}^{\Phi}(\Omega)$ is convex.

On the other hand, suppose $\mathscr{H}_{+}^{\Phi}(\Omega)$ is convex. We want to prove $\Phi(r) \equiv C r^{2}$, or equivalently, $h(r) \equiv r \varphi^{\prime}(r)-\varphi(r) \equiv 0$. Without loss of generality, we suppose that $0 \in \Omega$, and therefore there exists an $a>0$, such that the ball $B_{a}=B_{a}(0) \subset \Omega$. We will prove that $h(r) \equiv 0$ in two steps.

Step I. First, we claim that $h$ does not change its sigh in $[0,+\infty)$.

To prove this, suppose that $r_{0}>0$ satisfies $h\left(r_{0}\right)>0$. Then, by the continuity of $h$, there exists an $\varepsilon \in\left(0, r_{0}\right)$, such that

$$
h(r)>0, \quad \forall r \in\left(r_{0}-\varepsilon, r_{0}+\varepsilon\right) .
$$

Let

$$
u_{1}(x)=-\frac{x_{1}^{2}-x_{2}^{2}}{L}+r_{0} x_{1}+C_{1}, \quad x=\left(x_{1}, x_{2}, \ldots, x_{n}\right) \in \Omega,
$$

where $L$ and $C_{1}$ are two large positive numbers, such that

$$
\left|\nabla u_{1}(x)\right| \in\left(r_{0}-\varepsilon, r_{0}+\varepsilon\right), \quad \forall x \in \Omega,
$$

and $u_{1}(x) \geq 1$, for all $x \in \Omega$. 
Since $u_{1} \in C^{2}(\Omega),\left|\nabla u_{1}\right| \neq 0$ (see (4.8)), we have

$$
-\Delta_{\Phi} u_{1}=-h\left(\left|\nabla u_{1}\right|\right) \frac{\left(D^{2} u_{1} D u_{1}, D u_{1}\right\rangle}{\left|\nabla u_{1}\right|^{3}}-\varphi\left(\left|\nabla u_{1}\right|\right) \frac{\Delta u_{1}}{\left|\nabla u_{1}\right|} .
$$

It is easy to verify that

$$
\begin{aligned}
& \Delta u_{1}=0, \quad \text { in } \Omega, \\
& \left\langle D^{2} u_{1} D u_{1}, D u_{1}\right\rangle<0, \quad \text { in } \Omega \text {. }
\end{aligned}
$$

Thus, $u_{1} \in \mathscr{S}_{+}^{\Phi}(\Omega)$.

Let $v_{M} \in \mathscr{H}_{+}^{\Phi}(\Omega)$ be the solution of the following equation

$$
\left\{\begin{array}{l}
-\Delta_{\Phi} v_{M}=M, \quad \text { in } \Omega ; \\
\left.v_{M}\right|_{\partial \Omega}=0 .
\end{array}\right.
$$

We can prove that, if we choose $M$ sufficiently large, then $v_{M} \geq \sup _{x \in B_{a}} u_{1}(x)$, in $B_{a}$.

By Lemma $3.3(v), u_{1} \wedge v_{M} \in \mathscr{H}_{+}^{\Phi}(\Omega)$. Since $0 \in \mathscr{H}_{+}^{\Phi}(\Omega)$ and $\mathscr{H}_{+}^{\Phi}(\Omega)$ is convex, we have $t\left(u_{1} \wedge v_{M}\right) \in \mathscr{H}_{+}^{\Phi}(\Omega)$, for $t \in(0,1)$. Noting that

$$
t\left(u_{1} \wedge v_{M}\right)=t u_{1}, \quad \text { in } B_{a}, \forall t \in(0,1),
$$

we have

$$
0 \leq-\Delta_{\Phi}\left(t u_{1}\right), \quad \text { in } B_{a}, \forall t \in(0,1),
$$

that is, (see (4.9) and (4.10)),

$$
0 \leq-h\left(t\left|\nabla u_{1}\right|\right) \frac{\left\langle D^{2} u_{1} D u_{1}, D u_{1}\right\rangle}{\left|\nabla u_{1}\right|^{3}}, \quad \text { in } B_{a}, \forall t \in(0,1) .
$$

Therefore, from (4.8) and (4.11), we get $h(r) \geq 0$, for all $r \in\left(0, r_{0}\right)$.

Similarly, if $h\left(r_{0}\right)<0$ for some $r_{0}>0$, then $h(r) \leq 0$, for all $r \in\left(0, r_{0}\right)$. Therefore, we must have

$$
h(r) \geq 0, \quad \forall r \geq 0,
$$

or

$$
h(r) \leq 0, \quad \forall r \geq 0 .
$$

Step II. By what we established in the first step, we can suppose that (4.14) holds without loss of generality. We claim that $h \equiv 0$. Otherwise, there exists an $r_{0}>0$, such that $h\left(r_{0}\right)>0$.

Let $u_{1}$ be defined by (4.7). Since $h \geq 0$, we have (see (4.9)-(4.11))

$$
-\Delta_{\Phi}\left(t L u_{1}\right) \geq 0, \quad \text { in } \Omega, \forall t>0 .
$$


Let $u_{2}(x)=-r_{0} x_{1}+C_{2}$, such that $u_{2} \geq 1$, in $\Omega$. Then

$$
-\Delta_{\Phi}\left(t L u_{2}\right)=0, \quad \text { in } \Omega, \forall t>0 .
$$

Let $M$ be large enough, and $v_{M}$ be defined by (4.12), such that

$$
v_{M} \geq\left(L r_{0} / a\right) \max \left(u_{1}, u_{2}\right), \quad \text { in } B_{a} .
$$

Let $w_{i} \equiv\left(L r_{0} u_{i} / a\right) \wedge v_{M}, i=1,2$. Then $w_{i}=\left(L r_{0} / a\right) u_{i}$, in $B_{a}, i=1,2$, and by Lemma $3.3(\mathrm{v}), w_{i} \in \mathscr{H}_{+}^{\Phi}(\Omega), i=1,2$. Therefore,

and

$$
w \triangleq \frac{w_{1}+w_{2}}{2}=\frac{r_{0}}{2 a}\left(-x_{1}^{2}+x_{2}^{2}\right), \quad \text { in } B_{a},
$$

$$
|\nabla w|=\frac{r_{0}}{a} \sqrt{x_{1}^{2}+x_{2}^{2}}, \quad \text { in } B_{a} .
$$

Since $h$ is continuous, there exists an $\varepsilon \in\left(0, r_{0}\right)$, such that

$$
h(r)>0, \quad \forall r \in\left(r_{0}-\varepsilon, r_{0}+\varepsilon\right) .
$$

Noting that

$$
-\Delta_{\Phi} w=-h(|\nabla w|) \frac{-x_{1}^{2}+x_{2}^{2}}{\left(x_{1}^{2}+x_{2}^{2}\right)^{3 / 2}}, \quad \text { in } B_{a}
$$

and

$$
h(|\nabla w|)=h\left(\frac{r_{0}}{a} \sqrt{x_{1}^{2}+x_{2}^{2}}\right)>0, \quad \text { in }\left\{x \in B_{a} \mid \sqrt{x_{1}^{2}+x_{2}^{2}}>\frac{r_{0}-\varepsilon}{r_{0}} a\right\},
$$

we see that $\left\{x \in B_{a} \mid-\Delta_{\Phi} w(x)<0\right\}$ has positive measure. That is, $w \notin \mathscr{H}_{+}^{\Phi}(\Omega)$, contradicting the assumption. Therefore $h \equiv 0$ and consequently, $\Phi(r) \equiv C r^{2}$.

\section{Weak closeness of $\mathscr{H}_{+}^{\Phi}(\Omega)$}

It is easy to prove that $\mathscr{H}_{+}^{\Phi}(\Omega)$ is strongly closed in $W_{0}^{1, \Phi}(\Omega)$. If $\mathscr{H}_{+}^{\Phi}(\Omega)$ is convex, then it is also weakly closed in $W_{0}^{1, \Phi}(\Omega)$ by Mazur's Theorem. Theorem 4.3 shows that when $n \geq 2$ and $\Phi(r) \not \equiv C r^{2}, \mathscr{H}_{+}^{\Phi}(\Omega)$ is not convex. But we will prove that $\mathscr{H}_{+}^{\Phi}(\Omega)$ is still weakly closed in $W_{0}^{1, \Phi}(\Omega)$. More precisely, we have the following theorem.

THEOREM 5.1. Let $\Omega$ be a bounded Lipschitz domain, $\Phi$ satisfy (S1)-(S3) and $\Delta_{2-}$ condition. Suppose that $u_{k} \in \mathscr{H}_{+}^{\Phi}(\Omega)$ is bounded in $W_{0}^{1, \Phi}(\Omega)$, and $u_{k} \rightarrow u$, weakly in $W_{0}^{1,1}(\Omega)$. Then $u \in \mathscr{H}_{+}^{\Phi}(\Omega)$. 
ProOF. By the assumption of the theorem, we can suppose that $u_{k} \rightarrow u$, strongly in $L^{1}(\Omega)$. Thus, we can further suppose that

$$
u_{k} \rightarrow u, \quad \text { a.e. } \Omega \text {. }
$$

Therefore,

$$
u(x)=\liminf _{k \rightarrow+\infty} u_{k}(x)=\lim _{k \rightarrow+\infty} \inf _{j \geq k} u_{j}(x), \quad \text { a.e. } x \in \Omega
$$

Denote $y_{k}(x) \equiv \inf _{j \geq k} u_{j}(x)$. Then

$$
y_{k} \nearrow \text { u, a.e. } \Omega \text {. }
$$

Moreover, by Lemma $3.3(v), y_{k} \in \mathscr{H}_{+}^{\Phi}(\Omega)$ and

$$
\int_{\Omega} \Phi\left(\left|\nabla y_{k}\right|\right) d x \leq \int_{\Omega} \Phi\left(\left|\nabla u_{k}\right|\right) d x \leq C .
$$

Thus, by Lemma 2.3 and (5.3), we must have $y_{k} \rightarrow u$, weakly in $W_{0}^{1,1}(\Omega)$. Using Lemma 2.3 again, we get

$$
\int_{\Omega} \Phi(|\nabla u|) d x \leq \liminf _{k \rightarrow+\infty} \int_{\Omega} \Phi\left(\left|\nabla y_{k}\right|\right) d x .
$$

Since $y_{k} \in \mathscr{H}_{+}^{\Phi}(\Omega), T_{\Phi}\left(y_{k}\right)=y_{k}$ by Lemma 3.3 (iii). By (5.3), $\mathbb{K}(u) \subseteq \mathbb{K}\left(y_{k}\right)$. Thus, we get, from (3.4), that

$$
\int_{\Omega} \Phi\left(\left|\nabla y_{k}\right|\right) d x \leq \int_{\Omega} \Phi(|\nabla v|) d x, \quad \forall v \in \mathbb{K}(u) .
$$

Combining (5.5) with (5.4), we have

$$
\int_{\Omega} \Phi(|\nabla u|) d x \leq \int_{\Omega} \Phi(|\nabla v|) d x, \quad \forall v \in \mathbb{K}(u),
$$

that is, $u=T_{\Phi}(u)$. Consequently, $u \in \mathscr{H}_{+}^{\Phi}(\Omega)$.

When $\Omega$ is only a bounded domain, if $W_{0}^{1, \Phi}(\Omega)$ is reflexive, then Theorem 5.1 still holds. In general, we have:

THEOREM 5.2. Let $\Omega$ be a bounded domain, $\Phi$ satisfy (S1)-(S3) and $\Delta_{2}$-condition. Suppose that $u_{k} \in \mathscr{S}_{+}^{\Phi}(\Omega) \cap W_{0}^{1,1}(\Omega)$ is bounded in $W^{1, \Phi}(\Omega)$, and $u_{k} \rightarrow u$, weakly in $W_{0}^{1,1}(\Omega)$. Then $u \in \mathscr{S}_{+}^{\Phi}(\Omega) \cap W_{0}^{1,1}(\Omega)$. 
Note that $\mathscr{S}_{+}^{\Phi}(\Omega) \cap W_{0}^{1,1}(\Omega)=\mathscr{H}_{+}^{\Phi}(\Omega)$ if $\Omega$ is a Lipschitz domain, Theorem 5.1 is a special case of Theorem 5.2.

Theorem 5.1 implies that $\mathscr{H}_{+}^{\Phi}(\Omega)$ is weakly closed in $W_{0}^{1, \Phi}(\Omega)$. In fact, when $W_{0}^{1, \Phi}(\Omega)$ is reflexive, a bounded sequence converging weakly in $W_{0}^{1,1}(\Omega)$ must converge weakly in $W^{1, \Phi}(\Omega)$. Thus, at this time, Theorem 5.1 is equivalent to say that $\mathscr{H}_{+}^{\Phi}(\Omega)$ is weakly closed in $W_{0}^{1, \Phi}(\Omega)$. However, when $W_{0}^{1, \Phi}(\Omega)$ is not reflexive, a bounded sequence in it need not have a subsequence converging weakly. In addition, we do not know, in $W_{0}^{1, \Phi}(\Omega)$, if we can always say weak* convergence, since we do not know if $W_{0}^{1, \Phi}(\Omega)$ is the dual space of some normed linear space. Nevertheless, by Lemma 2.3, a bounded sequence in $W_{0}^{1, \Phi}(\Omega)$ has a subsequence converging weakly in $W_{0}^{1,1}(\Omega)$. Thus, in application, for example, when we treat some variational problems, we will find that Theorem 5.1 is more useful than the result of $\mathscr{H}_{+}^{\Phi}(\Omega)$ being weakly closed in $W_{0}^{1, \Phi}(\Omega)$.

A typical case we are interested in is when $\Phi(r) \equiv r^{p} / p$ for some $p \in(1,+\infty)$. At this time, $\Delta_{\Phi}$ is the so-called $p$-Laplacian and $\mathscr{H}_{+}^{\Phi}(\Omega)$ is denoted by $\mathscr{H}_{+}^{p}(\Omega)$. It is easy to verify that $\Phi$ satisfies $\Delta_{2}$-condition and $\nabla_{2}$-condition. Thus, $\mathscr{H}_{+}^{P}(\Omega)$ is convex if and only if $p=2$ or $n=1$. Moreover, it is weakly closed in $W_{0}^{1, p}(\Omega)$.

Another interesting case is when $\Phi(r) \equiv r \ln (1+r)$. Then, $\Delta_{\Phi}$ is called $L \ln L$ Laplacian and $\mathscr{H}_{+}^{\Phi}(\Omega)$ is denoted by $\mathscr{H}_{+}^{L \ln L}(\Omega)$. In this case, $\Phi$ satisfies $\Delta_{2-}$ condition. By Theorem 4.3, $\mathscr{H}_{+}^{L \ln L}(\Omega)$ is not convex when $n \geq 2$. By Theorem 5.1, $\mathscr{H}_{+}^{L \ln L}(\Omega)$ is weakly closed in $W_{0}^{1, L \ln L}(\Omega)$.

\section{Generalization}

In this section, we generalize the results obtained in Section 5. Let $G: \Omega \times \mathbb{R}^{n} \rightarrow \mathbb{R}$ be a measurable function satisfying

$$
\begin{gathered}
G(x, \cdot) \in C^{l}\left(\mathbb{R}^{n}\right) \cap C^{2}\left(\mathbb{R}^{n} \backslash\{0\}\right), \quad \forall x \in \Omega, \\
C_{1} \Phi(|\eta|) \leq G(x, \eta) \leq C_{2} \Phi(|\eta|), \quad \forall x \in \Omega, \eta \in \mathbb{R}^{n},
\end{gathered}
$$

and

$$
G_{\eta_{i} \eta_{j}}(x, \eta) \xi_{i} \xi_{j}>0, \quad \forall x \in \Omega, \eta, \xi \in\left(\mathbb{R}^{n} \backslash\{0\}\right),
$$

where $C_{2}>C_{1}>0$ are two constants.

Let $f \in W^{l . \Phi}(\Omega)$. Suppose that $b: \Omega \times \mathbb{R} \rightarrow \mathbb{R}$ is measurable in $x \in \Omega$ and continuous in $u \cdot \in \mathbb{R}$. Consider the following inequality

$$
\left\{\begin{array}{l}
-\operatorname{div}\left(G_{\eta}(x, \nabla u)\right) \geq b(x, u(x)), \quad \text { in } \Omega ; \\
\left.u\right|_{\partial \Omega}=f
\end{array}\right.
$$

We have 
THEOREM 6.1. Let $\Omega$ be a bounded Lipschitz domain, $\Phi$ satisfies (S1)-(S3) and $\Delta_{2}$-condition. Suppose that $G$ satisfies (6.1)-(6.3), $f \in W^{1, \Phi}(\Omega), b: \Omega \times \mathbb{R} \rightarrow \mathbb{R}$ is measurable in $x \in \Omega$ and continuous in $u \in \mathbb{R}$. Let $u_{k}(\cdot) \in W^{1, \Phi}(\Omega)$ satisfy (6.4) in the weak sense,

$$
u_{k} \rightarrow u, \text { weakly in } W^{1,1}(\Omega),
$$

and $u_{k}(\cdot)$ be bounded in $W^{1, \Phi}(\Omega)$. Moreover, suppose that $b\left(\cdot, u_{k}(\cdot)\right) \in L^{\Psi}(\Omega)$, and there exists a $b_{0}(\cdot) \in L^{\Psi}(\Omega)$, such that $b\left(x, u_{k}(x)\right) \geq b_{0}(x)$, a.e. $\Omega$, for all $k=1,2, \ldots$ Then $u \in W^{1, \Phi}(\Omega)$, and it satisfies $(6.4)$ in the weak sense too.

PROOF. We give a sketch of the proof.

If $b(x, u)$ is independent of $u$, then the result can be obtained by a modification of the proof of Theorem 5.1.

In general, by (6.5), we can suppose that $u_{k}(x)$ converges to $u(x)$ for almost all $x \in \Omega$. Thus, by Egorov's Theorem (see [7, Chapter 0], for example), for any $\varepsilon>0$, there exists a subset $E_{\varepsilon} \subset \Omega$, such that $\left|E_{\varepsilon}\right|<\varepsilon$ and $u_{k}(\cdot)$ converges to $u(\cdot)$ uniformly in $\Omega \backslash E_{\varepsilon}$, where $\left|E_{\varepsilon}\right|$ is the Lebesgue measure of $E_{\varepsilon}$. Let

$$
\tilde{b}_{\varepsilon}(x)= \begin{cases}b(x, u(x)), & \text { if } x \in \Omega \backslash E_{\varepsilon} \\ b_{0}(x), & \text { if } x \in E_{\varepsilon} .\end{cases}
$$

Then for any $\delta>0$, there exists a $K>0$ such that $b\left(x, u_{k}(x)\right) \geq \tilde{b}_{\varepsilon}(x)-\delta$, a.e. $x \in \Omega$, for all $k \geq K$. Thus, for any $k>K$,

$$
\left\{\begin{array}{l}
-\operatorname{div}\left(G_{\eta}\left(x, \nabla u_{k}\right)\right) \geq \tilde{b}_{\varepsilon}(x)-\delta, \quad \text { in } \Omega ; \\
\left.u_{k}\right|_{\partial \Omega}=f .
\end{array}\right.
$$

Consequently,

$$
\left\{\begin{array}{l}
-\operatorname{div}\left(G_{\eta}(x, \nabla u)\right) \geq \tilde{b}_{\varepsilon}(x)-\delta, \quad \text { in } \Omega \\
\left.u\right|_{\partial \Omega}=f .
\end{array}\right.
$$

Let $\delta \rightarrow 0^{+}$and $\varepsilon \rightarrow 0^{+}$, we get

$$
\left\{\begin{array}{l}
-\operatorname{div}\left(G_{\eta}(x, \nabla u)\right) \geq b(x, u(x)), \quad \text { in } \Omega ; \\
\left.u\right|_{\partial \Omega}=f .
\end{array}\right.
$$

\section{Acknowledgement}

The author would like to thank Professor Jiayu Li for many helpful suggestions and constant encouragement. 


\section{References}

[1] R. A. Adams, Sobolev spaces (Academic Press, New York, 1975).

[2] I. M. Gelfand and G. E. Shilov, Generalized functions, Vol. I (Academic Press, New York, 1964).

[3] J. L. Lions, Optimal control of systems governed by partial differential equations (Springer, New York, 1971).

[4] M. M. Rao and Z. D. Ren, Theory of Orlicz spaces (Marcel Dekker, New York, 1991).

[5] L. Schwartz, Théorie des distributions (Hermann, Paris, 1966).

[6] L. Simon, Lectures on geometric measure theory, Proceedings of the Centre for Mathematical Analysis ANU (Australian National University, Canberra, 1983).

[7] K. Yosida, Functional analysis, 6th edition (Springer, Berlin, 1980).

[8] A. Zygmund, Trigonometric series (Cambridge University Press, Cambridge, 1959).

\section{Department of Mathematics}

Fudan University

Shanghai 200433

China

e-mail: hwlou@fudan.edu.cn 